\title{
YAGO 4: A Reason-able Knowledge Base
}

\author{
Thomas Pellissier Tanon ${ }^{1(\bowtie)}$, Gerhard Weikum ${ }^{2}$, and Fabian Suchanek ${ }^{1}$ \\ 1 Télécom Paris, Institut Polytechnique de Paris, Palaiseau, France \\ thomas@pellissier-tanon.fr, suchanek@telecom-paris.fr \\ 2 Max Planck Institute for Informatics, Saarbrücken, Germany
}

\begin{abstract}
YAGO is one of the large knowledge bases in the Linked Open Data cloud. In this resource paper, we present its latest version, YAGO 4, which reconciles the rigorous typing and constraints of schema.org with the rich instance data of Wikidata. The resulting resource contains 2 billion type-consistent triples for 64 Million entities, and has a consistent ontology that allows semantic reasoning with OWL 2 description logics.
\end{abstract}

\section{Introduction}

A knowledge base $(\mathrm{KB})$ is a machine-readable collection of knowledge about the real world. A KB contains entities (such as organizations, movies, people, and locations) and relations between them (such as birthPlace, director, etc.). KBs have wide applications in search engines, question answering, fact checking, chatbots, and many other NLP and AI tasks. Numerous projects have constructed KBs automatically or by help of a community. Notable KBs include YAGO [17], DBpedia [1], BabelNet [14], NELL [2], KnowItAll [3], and Wikidata [18]. On the industry side, giants such as Amazon, Google, Microsoft, Alibaba, Tencent and others are running $\mathrm{KB}$ technology as a background asset, often referred to as knowledge graphs.

YAGO $[10,13,16,17]$ was one of the first academic projects to build a knowledge base automatically. The main idea of YAGO was to harvest information about entities from the infoboxes and categories of Wikipedia, and to combine this data with an ontological backbone derived from classes in WordNet [4]. Since Wikipedia is an excellent repository of entities, and WordNet is a widely used lexical resource, the combination proved useful. YAGO sent each fact through a pipeline of filtering, constraint checking, and de-duplication steps. This procedure scrutinized noisy input and boosted the quality of the final $\mathrm{KB}$, to a manually verified accuracy of $95 \%$. This precision was possible thanks to the tight control that the YAGO creators had over the extraction process, the filtering process, the ontological type system, the choice of the relations, and the semantic constraints. However, despite new versions YAGO2 and YAGO3 with substantial jumps in scope and size, the focus on Wikipedia infoboxes meant that YAGO has not arrived at the same scale as Freebase or Wikidata.

Meanwhile, Wikidata [18] has evolved into the world's foremost publicly available KB. It is a community effort where anybody can contribute facts - either

(C) Springer Nature Switzerland AG 2020

A. Harth et al. (Eds.): ESWC 2020, LNCS 12123, pp. 583-596, 2020.

https://doi.org/10.1007/978-3-030-49461-2_34 
by manually adding or curating statements in the online interface, or by bulkloading data. Wikidata has motivated more than 40,000 people who contribute at least once a month. The result is a public KB with $70 \mathrm{M}$ named entities, very good long-tail coverage, and impressive detail. ${ }^{1}$

At the same time, Wikidata understands itself as a collection of information, not as a collection of universally agreed-upon knowledge. It may intentionally contain contradictory statements, each with different sources or validity areas. Therefore, Wikidata does not enforce semantic constraints, such as "each person has exactly one father". Furthermore, the large user community has led to a proliferation of relations and classes: Wikidata contains $6.7 \mathrm{k}$ relations, of which only $2.6 \mathrm{~K}$ have more than 1000 facts, and it comprises around $2.4 \mathrm{M}$ classes $^{2}$, of which $80 \%$ have less than 10 instances. Many instances (e.g., all cities) are placed in the taxonomy under more than 60 classes, with three-fold multiple inheritance. This complexity is the trade-off that Wikidata has found to accommodate its large user community. For downstream applications, the convoluted and often confusing type system of Wikidata make browsing and question answering tedious. Moreover, there is little hope to run strict classical reasoners (e.g., for OWL 2) in a meaningful way, as the KB contains many small inconsistencies so that every possible statement is deducible regardless of whether it is intuitively correct or false. Some of these issues have been pointed out in the comprehensive study of KB quality by [19].

Example. To illustrate the shortcomings by the verbose and sometimes confusing type hierarchy of Wikidata, consider the entities Notre Dame de Paris (http:// www.wikidata.org/entity/Q2981) and Potala Palace (http://www.wikidata.org/ entity/Q71229) both landmarks of two world religions.

Notre Dame is an instance of types catholic cathedral and minor basilicas, with a rich set of superclasses. The Potala Palace in Lhasa is an instance of palace and tourist attraction. Interestingly, the latter does not have Notre Dame de Paris as an instance, neither directly nor indirectly. So a query for tourist attractions would find the Potala Palace but not Notre Dame.

Moreover, the class tourist attraction is a subclass of geographic object which is an instance of the class geometric concept which in turn has superclass mathematical concept. As a consequence, a query for mathematical concepts returns entities like tensor, polynomial, differential equation... and the Potala Palace as answers.

Contribution. In this resource paper, we describe the new YAGO version, YAGO 4, which aims to combine the best of the two worlds: It collects the facts about instances from Wikidata, but it forces them into a rigorous type hierarchy with semantic constraints. The complex taxonomy of Wikidata is replaced by

\footnotetext{
${ }^{1}$ All the numbers given in the paper about Wikidata are valid as of Feb. 24, 2020.

${ }^{2}$ Wikidata does not have a strong concept of a "class"; we use this term to denote entities that have superclasses (i.e., appear as left-hand argument of "subclass of" triples).
} 
the simpler and clean taxonomy of schema.org [8]. The classes are equipped with SHACL constraints [12] that specify disjointness, applicable relations, and cardinalities. This way, YAGO 4 transfers the rationale of the original YAGO from the combination of Wikipedia and WordNet to the combination of Wikidata and schema.org. The result is a new knowledge base, which is not just large, but also logically consistent, so that OWL-based reasoning is feasible. Hence we call YAGO 4 a "reason-able" knowledge base. The new resource is available at http://yago-knowledge.org under a permissive license (Creative Commons Attribution-ShareAlike). YAGO 4 also comes with a browser and a SPARQL endpoint. Figure 1 shows an excerpt of the new YAGO in the online browser.

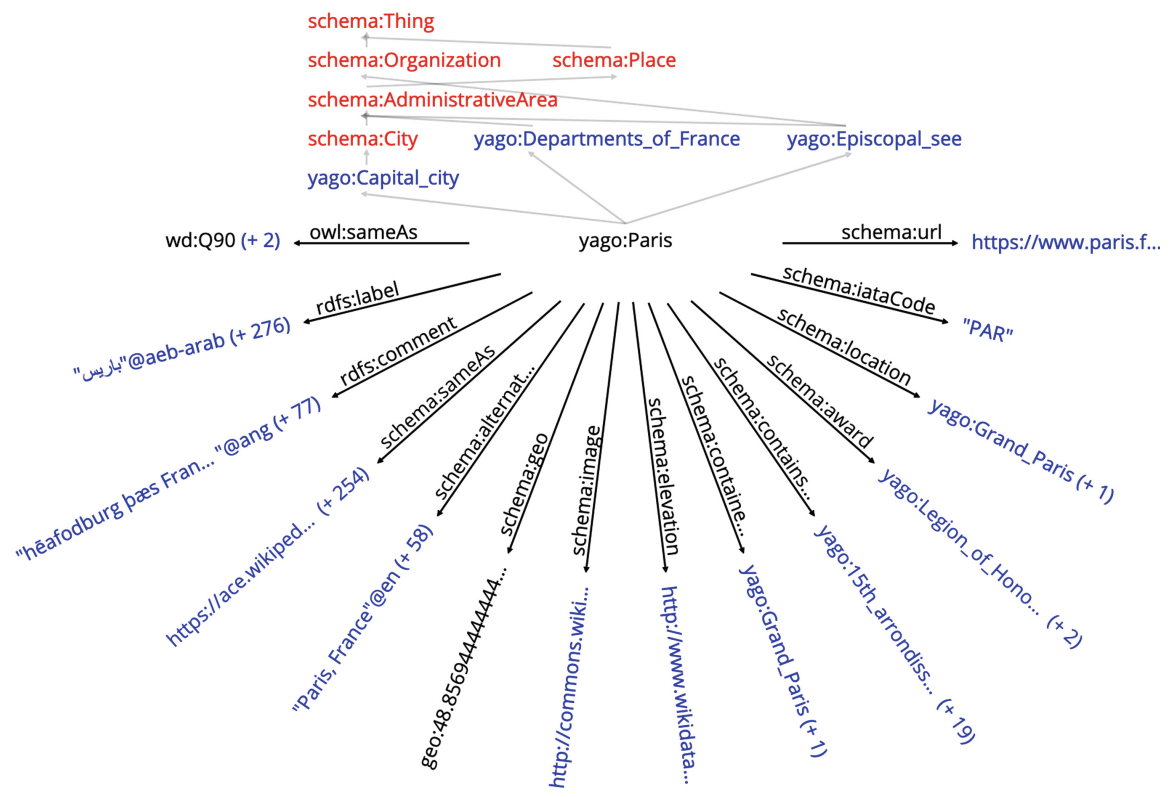

Fig. 1. The YAGO 4 Browser. Hovering reveals the full name of abbreviated items; all red and blue items are clickable. (Color figure online)

\section{Related Work}

The Linked Open data cloud contains several dozen general-purpose $\mathrm{KBs}^{3}$. YAGO 4 is not intended to replace these $\mathrm{KBs}$, but rather as an addition to this ecosystem with unique characteristics that complement the other players. For example, DBpedia also has a new version that ingests facts from Wikidata [11], with a well-designed pipeline that allows harvesting different knowledge sources [5]. This new DBpedia and YAGO 4 have made different design

\footnotetext{
$\overline{3}$ https://www.lod-cloud.net/.
} 
choices, resulting in different strengths and limitations. Our key priority has been to strengthen the logical rigor of the KB, so as to support OWL and other reasoners. This is why YAGO 4 builds on schema.org and adds its own constraint system which is much more elaborate than what DBpedia enforces.

\section{Design}

The construction of the YAGO 4 knowledge base is driven by several design decisions, which we explain and motivate next. The overarching point is to center YAGO 4 around a well-founded notion of classes. For example, a Person is defined as a subclass of Thing, and has an explicit set of possible relations such as birthDate, affiliation, etc. ${ }^{4}$ Conversely, other relations such as capitalOf, headquarter or population are not applicable to instances of the class Person. This overarching principle of semantic consistency unfolds into several design choices.

\subsection{Concise Taxonomy}

Wikidata contains a very detailed taxonomy to which the community contributes by adding instance Of and subclass Of statements. However, the resulting class hierarchy is so deep and convoluted that it is not easy to grasp and that browsing it is rather tedious. For example, Paris is an instance of 60 classes, 20 of which are called "unit", "entity", "subject", or "object". Moreover, the class hierarchy is not stable: any contributor can add or remove subclass Of links between any two classes. Potentially, this could lead to millions of entities being classified differently, just by a single edit. On the other hand, schema.org, the second major input to YAGO 4, has established itself as a reference taxonomy on the Web, beyond its initial aim at helping search engines to index web pages. It is stable, well maintained, and changes are made only by agreement in the W3C Schema.org Community Group ${ }^{5}$. At the same time, schema.org does not provide fine-grained classes such as "electric cars" or "villages" - which only Wikidata has. Schema.org also does not have any biochemical classes (such as proteins etc.).

We address the latter problem by using Bioschemas $[7]^{6}$. This project extends schema.org in the field of the life sciences - a field that is not covered in schema.org, and that is very prominent in Wikidata. We manually merged 6 Bioschemas classes into schema.org, referring to the merged taxonomy as the "schema.org taxonomy" for simplicity.

To obtain the stability of schema.org while preserving the fine-grained classes of Wikidata, we found the following solution: The top-level taxonomy of YAGO 4 is taken from schema.org (incl. Bioschemas), and leaf-level classes are taken from Wikidata. For this purpose, we manually mapped 235 classes of schema.org to Wikidata classes. Classes of schema.org that could not be mapped,

\footnotetext{
${ }^{4}$ For readability, we omit namespace prefixes in this paper.

${ }^{5}$ https://www.w3.org/community/schemaorg/.

${ }^{6}$ https://bioschemas.org.
} 
mostly shopping-related or social-media classes such as schema:LikeAction, were removed. With these inputs, the YAGO 4 taxonomy is then constructed as follows:

- For each instance in Wikidata, we consider each possible path in the Wikidata taxonomy to the root node. If the first class on the path has a Wikipedia article, we include it in YAGO 4. The rationale is that only classes with an English Wikipedia article are of sufficient interest for a wider audience and use cases.

- We then continue the path to the root in the Wikidata taxonomy, discarding all classes on the way, until we hit a class that has been mapped to schema.org. We continue our path to the root in the schema.org taxonomy, adding all classes on the way to YAGO 4.

- If we do not hit a class that has been mapped to schema.org, we discard the entire path. If an instance has no path with a class that qualifies for these criteria, we discard the instance.

We discard all Wikidata classes that have less than 10 direct instances. This threshold serves to ignore classes that have little value in use cases or are rather exotic. We further remove subclasses of a small list of meta-level Wikidata classes such as Wikipedia categories, disambiguation pages, etc. Finally, we drop subclasses of pair of classes for which we enforce disjointness constraints. These design choices allow us to model villages and cars, while significantly reducing the size of the taxonomy. From the $2.4 \mathrm{M}$ original Wikidata classes, we kept only 10k classes, shrinking the taxonomy by $99.6 \%$. We also discard $11 \mathrm{M}$ instances $(14 \%)$ - two thirds of which (7.5M) are Wikipedia-specific meta-entities (disambiguation page, category, wikitext template, etc.). Our strategy capitalizes on the stable backbone of schema.org, while being able to augment YAGO 4 with new data coming from Wikidata.

\subsection{Legible Entities and Relations}

YAGO 4 is stored in the RDF format. Unlike Wikidata, we chose to give humanreadable URIs to all entities, in order to make the $\mathrm{KB}$ more accessible for interactive use. If an entity has a Wikipedia page (which we know because Wikidata links it to Wikipedia), we take the Wikipedia title as the entity name. Otherwise, we concatenate the English label of the entity with its Wikidata identifier (e.g., Bischmisheim_Q866094). Studies like [15] suggest that the Wikidata labels are fairly stable, leading to fairly stable YAGO URIs. If the entity has no English label, we stay with the Wikidata identifier. We make the necessary changes to arrive at a valid local IRI name, and add the namespace of YAGO, http://yagoknowledge.org/resource/. This gives the vast majority of entities human-readable names, without introducing duplicates or ambiguity.

Wikidata has a very rich set of relations, but many of these have only very few facts. Indeed $61 \%$ of them have less than 1000 facts and $85 \%$ of them less than 10k. For YAGO 4, we chose to follow the successful model of previous YAGO 
versions, which have been parsimonious on the relations per class. We chose the relations from schema.org, which are each attached to a class. While these relations are conservative in coverage, they have emerged as a useful reference. We mapped 116 of these relations manually to the relations of Wikidata. We simply add this information to our schema, by using two new relations, yago:fromClass and yago:fromProperty, as shown here:

schema:Person yago:fromClass wd:Q215627

yago:birthPlaceProperty yago:fromProperty wdt:P569

The pipeline for $\mathrm{KB}$ construction takes care to implement these mappings (Sect.4.1). This process discards around 7k relations from Wikidata. As a byproduct, it gives human-readable names to all relations. Example relations are schema:birthPlace, schema:founder, and schema:containedInPlace. We use RDF and RDFS relations whenever possible, including rdfs:label and rdfs:comment instead of schema:name and schema:description. For example, the fact "wd:Q42 wdt:P31 wd:Q5" from Wikidata becomes

yago:Douglas_Adams rdf:type schema:Person

\subsection{Well-Typed Values}

YAGO 4 has not just well-typed entities, but also well-typed literals. For this purpose, we translate the data values of Wikidata to RDF terms. References to Wikidata entities are converted to references to the YAGO entities as explained in Sect.3.2. External URIs are converted into xsd:anyURI literals after normalizing them. ${ }^{7}$ We chose to keep external URIs as literals and not as entities, because we do not make any statements about URIs. Time values are converted to xsd:dateTime, xsd:date, xsd:gYearMonth or xsd:gYear, depending on the time precision. We discard the other time values whose precision could not be mapped to an XML schema type. Globe coordinates are mapped to schema:GeoCoordinates resources. Quantities are mapped to schema:QuantitativeValue resources (keeping the unit and precision). If there is no unit and an empty precision range, we map to xsd:integer where possible. If the unit is a duration unit (minutes, seconds...) and the precision range is empty, we map to xsd:duration. In this way, the vast majority of values are migrated to standard RDF typed literals.

\subsection{Semantic Constraints}

YAGO 4 has hand-crafted semantic constraints that not just keep the data clean, but also allow logical reasoning on the data. We model constraints in the W3C standards SHACL [12] and OWL. YAGO 4 currently has the following constraints:

\footnotetext{
${ }^{7}$ We follow the normalization suggested by RFC 2986 Section 6.2 .
} 
Disjointness. We specify 6 major top-level classes: schema:BioChemicalEntity, schema:Event, schema:Organization, schema:Person, schema:Place, and schema:CreativeWork. With the exception of schema:Organization/schema: Place, these are pairwise disjoint; so that these classes cannot have any instances in common. We use OWL to express, for example:

schema:Person owl:disjointWith schema:CreativeWork

Note that organizations are not disjoint from places, because many organizations are also located somewhere.

Domain and Range. Each relation comes with a domain and range constraint, meaning that a relation such as birthPlace can apply only to a person and a place. RDFS can specify the domain and range of relations by help of the predicates $r d f$ :domain and rdfs:range, but our constraints are different: If a KB contained the fact birthPlace (London, Paris), then the statement rdfs:domain (birthPlace,Person) would simply deduce that London must be a person. In contrast, our constraints would flag the KB as inconsistent. We use SHACL to express these constraints, as in this example:

schema:Person sh:property yago:birthPlaceProperty

yago:birthPlaceProperty sh:path schema:birthPlace

yago:birthPlaceProperty sh:node schema:Place

The same property can be used to describe entities of different classes. For example telephone can be used to describe both persons and organizations. In this case, the same property is going to be in the shapes of several classes. The domain of the property then is the union of all these classes.

In the same spirit, we also support disjunction in property ranges. For example, the range of author is Person union Organization. Following the same argument, the range of the birthDate property is the union of datatypes xsd:dateTime, xsd:date, xsd:gYearMonth and xsd:gYear to allow different calendar value precisions. Our range constraints also include the validation of xsd:string literals via regular expressions, as in this example:

schema:Person sh:property yago:telephoneProperty

yago:telephoneProperty sh:path schema:telephone yago:telephoneProperty sh:pattern " $+\backslash d\{1,3\} \ldots "$

Functional Constraints. A functional constraint says that a relation can have at most one object for a subject. Several of our relations are functional, e.g., birthPlace or gender. Again, we use SHACL:

yago:Person sh:property yago:birthPlaceProperty yago:birthPlaceProperty sh:maxCount "1" ^^xd:integer 
Cardinality Constraints. Going beyond functional constraints, we can also specify the maximal number of objects in general. For example, people can have only two parents in YAGO 4. We use again the SHACL sh:maxCount property.

YAGO 4 assumes that no other properties are allowed for each class, thereby interpreting the SHACL constraints under a "closed world assumption". The constraints are automatically enforced during the construction of the KB (see Sect.4.1), and so the data of YAGO 4 satisfies all constraints. Overall, the enforcement of constraints leads to the removal of $132 \mathrm{M}$ facts from Wikidata (i.e. $28 \%$ of all the facts). Since the constraints are enforced at KB-construction time, we can then add the deductive $r d f s$ :domain and $r d f s$ :range facts to YAGO 4 without risking that these deduce anything that violates the constraints.

The generated ontology uses the OWL 2 axioms DisjointClasses, ObjectPropertyDomain, DataPropertyDomain, ObjectPropertyRange, DataPropertyRange, ObjectUnionOf, FunctionalDataProperty, FunctionalObjectProperty, and falls into the OWL DL flavor. Statistics about the mapping and constraints are shown in Table 1.

Table 1. Schema and mapping statistics

\begin{tabular}{l|c}
\hline Item & Number \\
\hline Schema.org classes & 235 \\
\hline Bioschemas.org classes & 6 \\
\hline Object properties & 100 \\
\hline Datatype properties & 41 \\
\hline Node shapes & 49 \\
\hline Property shapes & 217 \\
\hline Domain constraints & 217 \\
\hline Object range constraints & 132 \\
\hline Datatype range constraints & 57 \\
\hline Regex constraints & 21 \\
\hline Disjoint constraints & 18 \\
\hline
\end{tabular}

\subsection{Annotations for Temporal Scope}

Following previous YAGO versions, YAGO 4 also attaches temporal information to its facts. We harvest these from the Wikidata qualifier system, which annotates facts with their validity time, provenance, and other meta information. We express the temporal scopes of facts by the relations schema:startDate and schema:endDate. Instead of relying on a custom format for these annotations, we made use of the RDF* model proposal [9], which has received good traction in recent years. For example, we state that Douglas Adams lived in Santa Barbara until 2001 as follows: 
$<<$ Douglas_Adams schema:homeLocation Santa_Barbara $>>$ schema:endDate 2001 We cannot use the usual Property Graph (PG) semantics of RDF*, because this would assert that Douglas Adams still lives in Santa Barbara. Rather, we use the "separate-assertions mode" (SA mode), which asserts only that he lived in Santa Barbara until 2001 - without saying where he currently lives.

\section{Knowledge Base}

\subsection{Construction}

We have designed a system that builds YAGO 4 automatically from (1) a Wikidata dump and (2) the SHACL shapes definitions of Sect. 3. We keep only the "truthy" Wikidata statements, i.e. for each subject and predicate we keep only the statements with the "best" rank (a.k.a. "preferred" if a statement with such a rank exists, "normal" if not).

The KB building system constructs the class hierarchy, the entities, and the facts as outlined in Sect. 3. Its main purpose is then to enforce the constraints (Sect. 3.4). If a resource is an instance of disjoint classes, we drop the two rdf:type relations leading to this conflict. We drop all instances that are not instances of any class. We enforce domain, range and regular-expression constraints by pruning all candidate facts that would violate a constraint. Finally, we check the cardinality constraints, removing all objects if there are too many for a given subject.

Our system is implemented in the Rust programming language ${ }^{8}$, using the Iterator infrastructure to ingest and output data streams. We use the already existing stream operators, which resemble those of relational algebra (map/project, filter, flat map, collect/materialize into a hash structure). We also implemented new operators particularly for YAGO 4 (stream-hash join, streamhash anti join, group-by, and transitive closure). For example, the owl:sameAs links between YAGO 4 and Freebase can be extracted from Wikidata by the following algebraic operator plan:

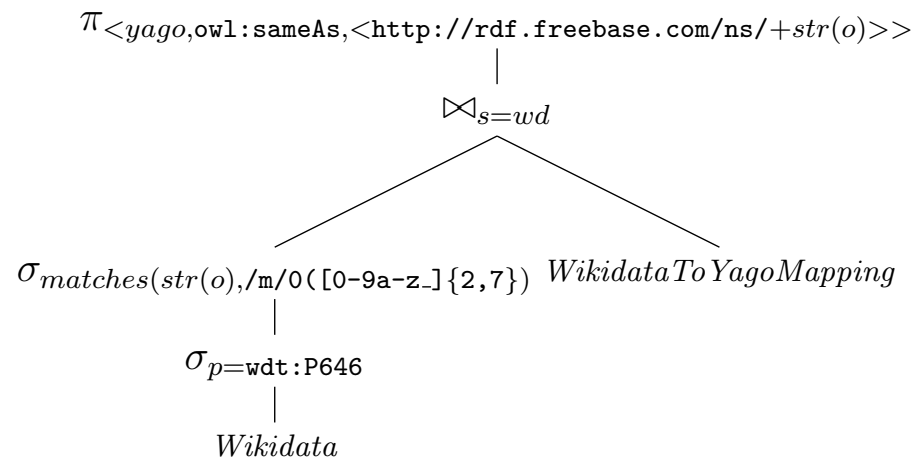

$\overline{8}$ https://www.rust-lang.org/. 
Here, $\pi$ is the projection operator, $\sigma$ the selection, $\bowtie$ the inner join, Wikidata the table of all Wikidata triples $(s, p, o)$, and WikidataToYagoMapping the mapping between Wikidata and YAGO instances (wd,yago). To avoid reading the full Wikidata N-Triples dump each time, we first load the Wikidata dump into the RocksDB key-value store to index its content ${ }^{9}$. This index allows for efficiently selecting triples based on a predicate or a (predicate, subject) tuple, and getting back a stream of triples from the database.

The advantage of having operator plans in Rust is that we can benefit from declarative programs where performance optimizations are carried out by the compiler, generating highly efficient native code. After having loaded the data into RocksDB, our execution plan generates the Wikipedia-flavored YAGO 4 (see below) in two hours on a commodity server.

We ran our system on a dump of $78 \mathrm{M}$ Wikidata items. $8 \mathrm{M}$ of these are entities about Wikimedia Websites-related entities, such as categories. From the $474 \mathrm{M}$ Wikidata facts whose property has been mapped to schema.org, we filtered out $89 \mathrm{M}$ of them because of the domain constraints and $42 \mathrm{M}$ more because of the range and regex constraints. The cardinality constraints lead to the removal of an extra $0.6 \mathrm{M}$ facts.

\subsection{Data}

YAGO 4 is made available in three "flavors":

- Full: This flavor uses all data from Wikidata, resulting in a very large KB.

- Wikipedia: This smaller flavor of YAGO 4 contains only the instances that have a Wikipedia article (in any language).

- English Wikipedia: This is an additional restriction of the Wikipedia flavor, containing only instances that have an English Wikipedia article.

All three flavors of YAGO 4 are built in the same way, and have the same schema, with 116 properties and the same taxonomy of 140 top-level classes from schema.org and bioschemas.org, and the same subset of Wikidata classes. Table 2 shows statistics for the three YAGO 4 variants, generated from the Wikidata N-Triples dump of November 25, 2019.

Each flavor of YAGO 4 is split into the following files:

- Taxonomy: The full taxonomy of classes.

- Full-types: All rdf:type relations.

- Labels: All entity labels (rdfs:label, rdfs:comment and schema: alternateName).

- Facts: The facts that are not labels.

- Annotations: The fact annotations encoded in RDF* [9].

- SameAs: The owl:sameAs links to Wikidata, DBpedia, and Freebase and the schema:sameAs to all the Wikipedias.

${ }^{9}$ https://rocksdb.org/. 
- Schema: The schema.org classes and properties, in OWL 2 DL.

- Shapes: The SHACL constraints used to generate YAGO 4.

Each file is a compressed N-Triples file, so that standard tools can directly ingest the data.

Table 2. Size statistics for YAGO 4 in the flavors Full, Wikipedia (W), and English Wikipedia (E), Wikidata and DBpedia (per DBpedia SPARQL server on 2020-03-04).

\begin{tabular}{l|l|l|l|l|l}
\hline & Yago Full & Yago W & Yago E & Wikidata & DBpedia \\
\hline Classes & 10124 & 10124 & 10124 & $2.4 \mathrm{M}$ & $484 \mathrm{k}$ \\
\hline Classes from Wikidata & 9883 & 9883 & 9883 & $2.4 \mathrm{M}$ & 222 \\
\hline Individuals & $67 \mathrm{M}$ & $15 \mathrm{M}$ & $5 \mathrm{M}$ & $78 \mathrm{M}$ & $5 \mathrm{M}$ \\
\hline Labels (rdfs:label) & $303 \mathrm{M}$ & $137 \mathrm{M}$ & $66 \mathrm{M}$ & $371 \mathrm{M}$ & $22 \mathrm{M}$ \\
\hline $\begin{array}{l}\text { Descriptions } \\
\text { (rdfs:comment) }\end{array}$ & $1399 \mathrm{M}$ & $139 \mathrm{M}$ & $50 \mathrm{M}$ & $2146 \mathrm{M}$ & $12 \mathrm{M}$ \\
\hline $\begin{array}{l}\text { Aliases } \\
\text { (schema:alternateName) }\end{array}$ & $68 \mathrm{M}$ & $21 \mathrm{M}$ & $14 \mathrm{M}$ & $71 \mathrm{M}$ & 0 \\
\hline $\begin{array}{l}\text { rdf:type (without transi- } \\
\text { tive closure) }\end{array}$ & $70 \mathrm{M}$ & $16 \mathrm{M}$ & $5 \mathrm{M}$ & $77 \mathrm{M}$ & $114 \mathrm{M}$ \\
\hline Facts & $343 \mathrm{M}$ & $48 \mathrm{M}$ & $20 \mathrm{M}$ & $974 \mathrm{M}$ & $131 \mathrm{M}$ \\
\hline $\begin{array}{l}\text { Avg. \# of facts per } \\
\text { entity }\end{array}$ & 5.1 & 3.2 & 4 & 12.5 & 26 \\
\hline sameAs to Wikidata & $67 \mathrm{M}$ & $15 \mathrm{M}$ & $5 \mathrm{M}$ & $\mathrm{N} . \mathrm{A}$ & $816 \mathrm{k}$ \\
\hline sameAs to DBpedia & $5 \mathrm{M}$ & $5 \mathrm{M}$ & $5 \mathrm{M}$ & 0 & $\mathrm{~N} . \mathrm{A}$. \\
\hline sameAs to Freebase & $1 \mathrm{M}$ & $1 \mathrm{M}$ & $1 \mathrm{M}$ & $1 \mathrm{M}$ & $157 \mathrm{k}$ \\
\hline \begin{tabular}{l} 
sameAs to Wikipedia \\
\hline Fact annotations
\end{tabular} & $43 \mathrm{M}$ & $43 \mathrm{M}$ & $26 \mathrm{M}$ & $66 \mathrm{M}$ & $13 \mathrm{M}$ \\
\hline \begin{tabular}{l} 
Dump size \\
\hline
\end{tabular} & $6.5 \mathrm{M}$ & $2.2 \mathrm{M}$ & $1.7 \mathrm{M}$ & $220 \mathrm{M}$ & 0 \\
\hline & $60 \mathrm{~GB}$ & $7 \mathrm{~GB}$ & $3 \mathrm{~GB}$ & $127 \mathrm{~GB}$ & $99 \mathrm{~GB}$ \\
\hline
\end{tabular}

\subsection{Access}

Web Page. The YAGO 4 knowledge base is available at http://yago-knowledge. org. The Web page offers an introduction to YAGO, documentation ("Getting started"), and a list of publications and contributors. The Web page also has a schema diagram that lists all top-level classes with their associated relations and constraints.

License. The entire YAGO 4 knowledge base, as well as all previous versions and the logo, can be downloaded from the Web page. YAGO 4 is available under a Creative Commons Attribution-ShareAlike License. The reason for this choice 
is that, while Wikidata is in the public domain, schema.org is under a Creative Commons Attribution-ShareAlike License. ${ }^{10}$

Source Code. We have released the source code for constructing YAGO 4 on GitHub at https://github.com/yago-naga/yago4 under the GNU GPL v3+ license.

SPARQL Endpoint. YAGO 4 comes with a responsive SPARQL endpoint, which can be used as an API or interactively. The URL is http://yago-knowledge.org/ sparql/query. The YAGO URIs are also all dereferencable, thus complying with the Semantic Web best practice.

Browser. YAGO 4 comes with a graphical KB browser, with an example shown in Fig. 1. For each entity, the browser visualizes the outgoing relationships in a star-shape around the entity. Above the entity, the browser shows the hierarchy of all classes of which the entity is a (transitive) instance, including those with multiple inheritance. If an entity has more than one object for a given relation, a relation-specific screen shows all objects of that relation for the entity. For size reasons, the browser shows only the Wikipedia flavor of YAGO.

Applications. YAGO has already been used in quite a number of projects [16], including question answering, entity recognition, and semantic text analysis. We believe that the new version of YAGO opens up the door to an entire array of new applications, because it is possible to perform logical reasoning on YAGO 4. Not only is the KB equipped with semantic constraints, but it is also provably consistent. We have checked the "English Wikipedia" flavor of YAGO 4 with the OWL 2 DL reasoner HermiT [6], proving its logical consistency. ${ }^{11}$ This makes it possible to perform advanced kinds of logical inference on YAGO 4.

\section{Conclusion}

This paper presents YAGO 4, the newest version of the YAGO knowledge base. The unique characteristics of YAGO 4 is to combine the wealth of facts from Wikidata with the clean and human-readable taxonomy from schema.org, together with semantic constraints that enforce logical consistency. This way, the resulting $\mathrm{KB}$ can be processed with OWL and other reasoners, and is also more user-friendly for browsing and question answering. We hope that the YAGO 4 resource fills a gap in the landscape of public KBs, and will be useful in downstream applications.

We plan to release updates of YAGO 4 to reflect the changes in Wikidata. A change of the schema vocabulary would require human intervention, and could

10 http://schema.org/docs/terms.html.

11 HermiT was unable to load the "Full" flavor due to a memory overflow, but it contains the same taxonomy and the same constraints as the "English Wikipedia" flavor. 
be done a few times a year. Future work includes extending the set of semantic constraints to capture inverse functions, symmetric and transitive properties, and more. We also consider tapping into additional data sources, beyond Wikidata, to further enrich the factual knowledge of YAGO 4.

Acknowledgements. This work was partially supported by the grant ANR-16-CE230007-01 ("DICOS").

\section{References}

1. Auer, S., Bizer, C., Kobilarov, G., Lehmann, J., Cyganiak, R., Ives, Z.: DBpedia: a nucleus for a web of open data. In: Aberer, K., et al. (eds.) ASWC/ISWC -2007. LNCS, vol. 4825, pp. 722-735. Springer, Heidelberg (2007). https://doi.org/10. 1007/978-3-540-76298-0_52

2. Carlson, A., Betteridge, J., Kisiel, B., Settles, B., Jr. Hruschka, E.R., Mitchell, T.M.: Toward an architecture for never-ending language learning. In: AAAI (2010). http://www.aaai.org/ocs/index.php/AAAI/AAAI10/paper/view/1879

3. Etzioni, O., et al.: Web-scale information extraction in knowitall. In: WWW, pp. 100-110 (2004). https://doi.org/10.1145/988672.988687

4. Fellbaum, C. (ed.): WordNet: An Electronic Lexical Database. MIT Press, Cambridge (1998). https://mitpress.mit.edu/books/wordnet

5. Frey, J., Hofer, M., Obraczka, D., Lehmann, J., Hellmann, S.: DBpedia FlexiFusion the best of Wikipedia > Wikidata > your data. In: Ghidini, C., et al. (eds.) ISWC 2019. LNCS, vol. 11779, pp. 96-112. Springer, Cham (2019). https://doi.org/10. 1007/978-3-030-30796-7_7

6. Glimm, B., Horrocks, I., Motik, B., Stoilos, G., Wang, Z.: HermiT: An OWL 2 Reasoner. J. Autom. Reasoning 53(3), 245-269 (2014). https://doi.org/10.1007/ s10817-014-9305-1

7. Gray, A.J.G., Goble, C.A., Jimenez, R.: Bioschemas: from potato salad to protein annotation. In: ISWC (2017). http://ceur-ws.org/Vol-1963/paper579.pdf

8. Guha, R.V., Brickley, D., Macbeth, S.: Schema.org: evolution of structured data on the web. Commun. ACM 59(2), 44-51 (2016). https://doi.org/10.1145/2844544

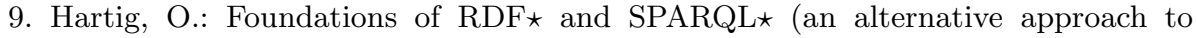
statement-level metadata in RDF). In: Alberto Mendelzon Workshop on Foundations of Data Management and the Web (2017). http://ceur-ws.org/Vol-1912/ paper12.pdf

10. Hoffart, J., Suchanek, F.M., Berberich, K., Lewis-Kelham, E., de Melo, G., Weikum, G.: YAGO2: exploring and querying world knowledge in time, space, context, and many languages. In: WWW, pp. 229-232 (2011). https://doi.org/10. $1145 / 1963192.1963296$

11. Ismayilov, A., Kontokostas, D., Auer, S., Lehmann, J., Hellmann, S.: Wikidata through the eyes of dbpedia. Semant. Web 9(4), 493-503 (2018). https://doi.org/ 10.3233/SW-170277

12. Knublauch, H., Kontokostas, D.: Shapes constraint language (SHACL). W3C Candidate Recommendation, 11(8) (2017). https://www.w3.org/TR/shacl/

13. Mahdisoltani, F., Biega, J., Suchanek, F.M.: YAGO3: a knowledge base from multilingual Wikipedias. In: CIDR (2015). http://cidrdb.org/cidr2015/Papers/CIDR15 Paper1.pdf 
14. Navigli, R., Ponzetto, S.P.: Babelnet: Building a very large multilingual semantic network. In: ACL, pp. 216-225 (2010). https://www.aclweb.org/anthology/P10$1023 /$

15. Pellissier Tanon, T., Kaffee, L.: Property label stability in wikidata: evolution and convergence of schemas in collaborative knowledge bases. In: WikiWorkshop, WWW, pp. 1801-1803 (2018). https://doi.org/10.1145/3184558.3191643

16. Rebele, T., Suchanek, F.M., Hoffart, J., Biega, J., Kuzey, E., Weikum, G.: YAGO: a multilingual knowledge base from Wikipedia, Wordnet, and Geonames. In: ISWC, pp. 177-185 (2016). https://doi.org/10.1007/978-3-319-46547-0_19

17. Suchanek, F.M., Kasneci, G., Weikum, G.: YAGO: a core of semantic knowledge. In: WWW, pp. 697-706 (2007). https://doi.org/10.1145/1242572.1242667

18. Vrandecic, D., Krötzsch, M.: Wikidata: a free collaborative knowledgebase. Commun. ACM 57(10), 78-85 (2014). https://doi.org/10.1145/2629489

19. Zaveri, A., Rula, A., Maurino, A., Pietrobon, R., Lehmann, J., Auer, S.: Quality assessment for linked data: a survey. Semant. Web 7(1), 63-93 (2016). https://doi. org/10.3233/SW-150175 